\title{
Performance of the Vitek 2 system software version 5.03 in the bacterial identification and antimicrobial susceptibility test: evaluation study of clinical and reference strains of Gram-positive cocci
}

\author{
Thiago Galvão da Silva Paim ${ }^{[1]}$, Vlademir Vicente Cantarelli ${ }^{[1]}$ and Pedro Alves d'Azevedo ${ }^{[1]}$
}

[1]. Laboratório de Cocos Gram-Positivos, Universidade Federal de Ciências da Saúde de Porto Alegre, Porto Alegre, RS.

\begin{abstract}
Introduction. The genera Enterococcus, Staphylococcus and Streptococcus are recognized as important Gram-positive human pathogens. The aim of this study was to evaluate the performance of Vitek 2 in identifying Gram-positive cocci and their antimicrobial susceptibilities. Methods. One hundred four isolates were analyzed to determine the accuracy of the automated system for identifying the bacteria and their susceptibility to oxacillin and vancomycin. Results. The system correctly identified $77.9 \%$ and $97.1 \%$ of the isolates at the species and genus levels, respectively. Additionally, $81.8 \%$ of the Vitek 2 results agreed with the known antimicrobial susceptibility profiles. Conclusion. Vitek 2 correctly identified the commonly isolated strains; however, the limitations of the method may lead to ambiguous findings.
\end{abstract}

Keywords: Gram-positive cocci. Vitek 2 automated system.

Gram-positive cocci are widely distributed as part of the normal flora in humans; however, some species are recognized as major human pathogens and cause a large variety of infections worldwide. These microorganisms are frequently isolated from bloodstream infections, skin and soft tissue infections, sepsis, urinary tract infections and lower respiratory tract infections ${ }^{1}$.

Automated bacterial identification in the clinical laboratory provides a rapid and reliable diagnosis for most pathogens involved in infectious diseases. A previous study demonstrated the satisfactory performances of the automated methodologies, resulting in their use in routine practice with a highly acceptable level of identification accuracy; additionally, automated identification enabled the interpretation of antimicrobial susceptibility tests for the correct treatment of patients ${ }^{2}$.

The aim of the present study was to evaluate the performance of the Vitek 2 automated system in the identification of bacteria and antimicrobial susceptibilities of Gram-positive cocci isolates recovered from clinical samples and reference strains.

The study was performed at Laboratório de Cocos GramPositivos (LCGP) of the Universidade Federal de Ciências da Saúde de Porto Alegre (UFCSPA) and Laboratório Qualità,

\footnotetext{
Address to: Ms. Thiago Galvão da Silva Paim. Universidade Federal de Ciências da Saúde de Porto Alegre. Rua Sarmento Leite 245, 90050-170 Porto Alegre, RS, Brasil.

Phone: 5551 3303-8742; Fax: 5551 3303-8810

e-mail: thiagopucrs@gmail.com

Received 12 June 2013

Accepted 19 November 2013
}

Rio Grande do Sul, Brazil. The isolates included in the present study were selected from the strain collection belonging LCGP. A total of 104 isolates of Gram-positive cocci were analyzed, including 29 reference strains selected from the American Type Culture Collection (ATCC) and 75 clinical strains isolated from different patients; these strains included Staphylococcus coagulase-negative $(\mathrm{n}=36)$, Enterococcus spp. $(\mathrm{n}=33)$ and Staphylococcus aureus $(\mathrm{n}=6)$. All of these strains have been previously characterized by the LCGP with regard to their virulence factors using molecular methods and susceptibility profiles and were identified at the species level using conventional reference methods ${ }^{3,4}$. For the identification of staphylococci, the following characteristics were tested: catalase; colony morphology and pigmentation; Gram stain; hemolysis; susceptibility to novobiocin; polymyxin B; fosfomycin and deferoxamine; enzyme activity of arginine arylamidase; ornithine decarboxylase and urease; and acid production from trehalose, mannitol, mannose, xylose, cellobiose, arabinose, maltose, lactose, sucrose and raffinose. For the enterococcal isolates, the following phenotypic characteristics were evaluated: catalase; colony morphology and pigmentation; esculin hydrolysis in the presence of $40 \%$ bile; growth in $6.5 \% \mathrm{NaCl}$; motility; and acid production from mannitol, sorbose, arginine, arabinose, sorbitol, raffinose, sucrose, pyruvate and methyl-glucopyranoside (MGP). Susceptibility to oxacillin and vancomycin was evaluated using the broth microdilution and disk-diffusion reference methods according the Clinical and Laboratory Standards Institute (CLSI) documents M7-A6 $6^{5}$ and M100-S2 $1^{6}$, respectively. Vitek 2 (bioMérieux, Marcy L'E`toile, France) bacterial identification and antimicrobial susceptibility testing (AST) methods were evaluated according to the manufacturer's instructions. 
The comparative analysis of the bacterial identification by Vitek 2 and the conventional methods was performed, and the accuracy (percentage of matched identification) was characterized. The AST results evaluated using the Vitek 2 automated system were compared to those obtained using the reference method, and the agreement rates were analyzed. The type of error was evaluated using the following criteria: I) very major error (VME) defined as a resistant bacterial isolate appearing susceptible by Vitek 2; II) major error (ME) defined as a susceptible isolate displaying a resistant profile by Vitek 2; or III) minor error (MiE) defined as microorganisms for which the AST reference indicated intermediate resistance and susceptibility or resistance by an automated AST test.

The Vitek 2 system correctly identified 81 (77.9\%) and $101(97.1 \%)$ of the 104 bacterial isolates at the species and genus levels, respectively. Additionally, Staphylococcus aureus was identified more quickly than the other staphylococcal isolates $(\mathrm{p}<0.05)$ (Table 1).

The Vitek 2 automated method was accurate for specieslevel identification of the commonly isolated Gram-positive cocci. All of the clinical isolates of $S$. aureus and Enterococcus faecalis were correctly identified. The bacterial isolates were misidentified or showed low-level discrimination in the clinical samples of Staphylococcus epidermidis (25\%), Staphylococcus haemolyticus (66.7\%), Staphylococcus hominis (16.7\%), Staphylococcus saprophyticus and Staphylococcus warneri (both 20\%). In the Enterococcus strains, Enterococcus avium and Enterococcus durans were misidentified as other enterococcal isolates, and the discordant results were found $50 \%$ of Enterococcus casseliflavus, $75 \%$ of Enterococcus faecium and 25\% of Enterococcus gallinarum isolates. Among the 29 reference strains, a definitive species identification was provided by the automated system for $22(75.9 \%)$ strains. The incorrect identification or low-level discrimination results are listed in Table 2. The minimal inhibitory concentrations (MICs) of oxacillin and vancomycin generated by the automated system were compared according to the error type, and the results are shown in the Table 3. In total, 45 of the 55 (81.8\%) bacterial isolates agreed with the identifications made using the conventional antimicrobial susceptibility profile.

At the species level, the automated Vitek 2 system was able to identify the commonly isolated staphylococci and enterococci strains, such as $S$. aureus and E. faecalis, respectively, and all of these isolates, as well as the streptococci reference strains, were correctly identified. These results were in agreement with those reported by Chatzigeorgiou ${ }^{7}$ et al., who evaluated the performance of the Vitek 2 system in comparison with other automated systems ${ }^{7}$. However, in the present study, the Vitek 2 version 5.03 software was not able to correctly identify all of the $S$. epidermidis isolates, with only $71.4 \%$ of the isolates matched at the species level.

S. haemolyticus is frequently isolated from blood cultures and has a tendency to develop resistance to multiple antimicrobial drugs $^{8}$, similar to $S$. warneri, which is another CoNS that can cause catheter-related bacteremia and native and prosthetic valve endocarditis 9 . Two isolates of this bacterial species were misclassified as $S$. warneri by the Vitek 2 system.

Two S. saprophyticus were misidentified as $S$. warneri and $S$. cohnii subs. urealyticus by the Vitek 2 automated system. According to the biochemical profile of these isolates, major error in the bacterial identification can be evaluated by novobiocin susceptibility, as $S$. saprophyticus and $S$. cohnii are resistant, while $S$. warneri is susceptible ${ }^{3}$. Moreover, S. saprophyticus and S. cohnii subs. urealyticus have similar

TABLE 1 - Descriptive measures from the automated system Vitek 2 compact.

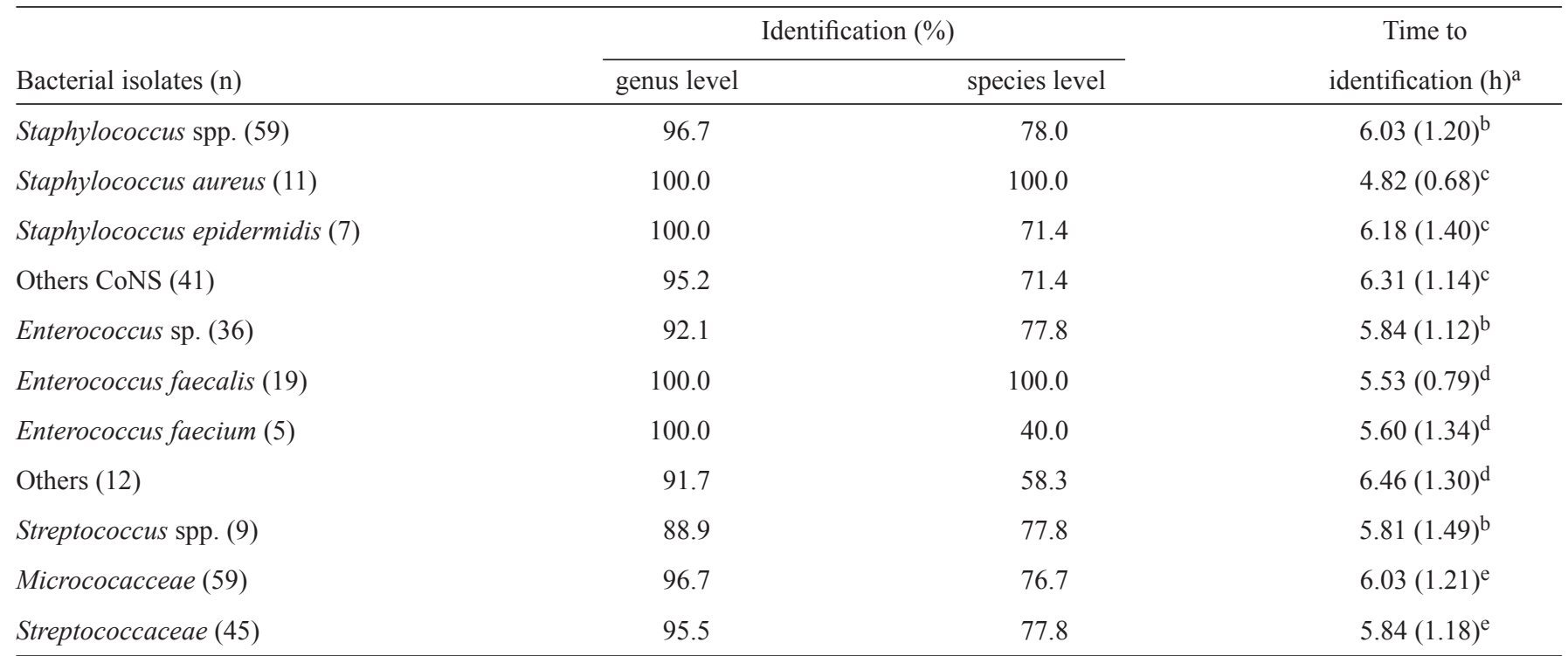

a mean (SD - standard deviation); analysis of variance (ANOVA) followed by Student-Newman-Keuls post hoc test. The data were tested for normal distributions

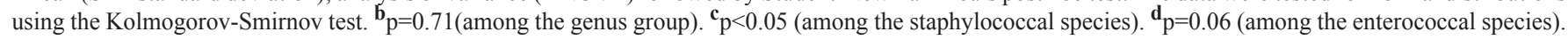

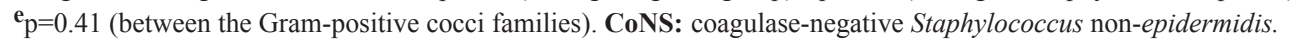


TABLE 2 - Bacterial species reported by the Vitek 2 System that were misidentified or showed low discrimination.

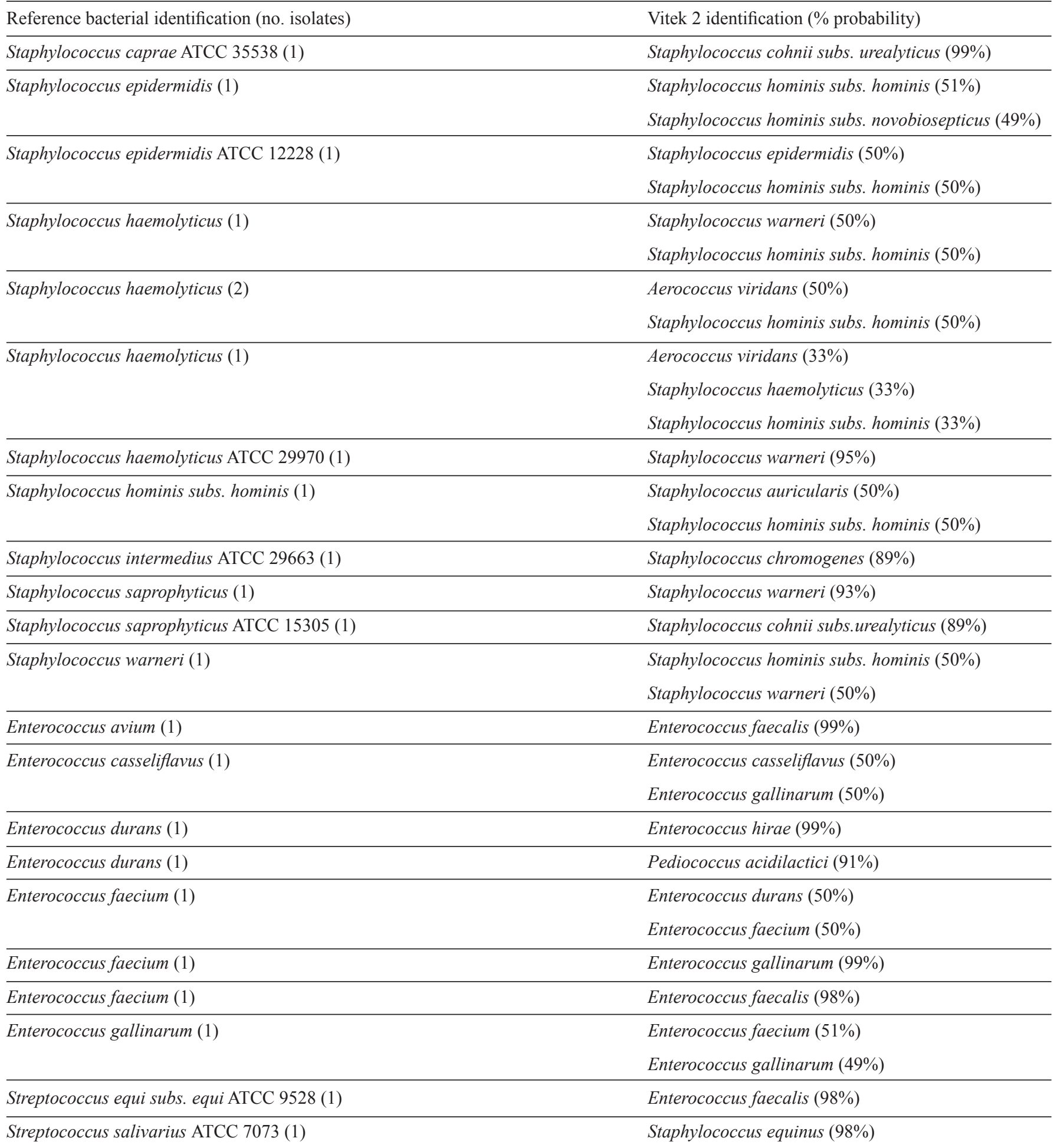

Subs: subspecie. 
TABLE 3 - Agreement of the antimicrobial susceptibility tests and the type of error among the staphylococci and enterococci isolates. ${ }^{\text {a }}$

\begin{tabular}{|c|c|c|c|c|c|c|c|}
\hline \multirow[b]{2}{*}{ Isolates (n) } & \multicolumn{2}{|c|}{ Agreements } & \multicolumn{2}{|c|}{ Errors } & \multicolumn{3}{|c|}{ Type of error (n) } \\
\hline & $\mathrm{n}$ & $\%$ & $\mathrm{n}$ & $\%$ & VME & ME & $\mathrm{MiE}$ \\
\hline Staphylococci (33) & 28 & 84.8 & 5 & 15.2 & 3 & 2 & 0 \\
\hline Enterococci $(22)^{\mathrm{c}}$ & 17 & 77.3 & 5 & 22.7 & 1 & 4 & 0 \\
\hline
\end{tabular}

aStreptococci reference strains were not tested. ${ }^{\mathbf{b}}$ Susceptibility to oxacillin. ${ }^{\mathbf{c}}$ Susceptibility to vancomycin. VME: very major error; ME: major error; MiE: minor error.

phenotypic profiles and exhibit low discriminatory power in bacterial identification; both species are urease positive and novobiocin resistant. The clinical laboratory exams should be able to differentiate these strains, as $S$. cohnii subs. urealyticus is recognized as a pathogen in infectious diseases, such as endocarditis, septicemia and urinary tract infections; however, previous study have reported difficulty in routinely identifying this microorganism ${ }^{10}$.

In the present study, the agreement identification rate of the enterococci isolates was similar to a previous study, and all of the $E$. faecalis isolates were correctly identified ${ }^{11}$. However, 3 of the 5 E. faecium strains were misclassified or showed low-level discrimination. The bacterial identification of the Enterococcus isolates is important because this genus includes some of the most important multidrug-resistant organisms in healthcare-associated infections. These isolates usually affect patients who are debilitated by other concurrent illnesses or are undergoing prolonged hospitalization. Including the E. faecium strains, these pathogens have the ability to succeed in the hospital environment ${ }^{12}$.

The automated system was able to identify 11 Enterococcus, 26 Staphylococcus and 37 Streptococcus species and subspecies using the Gram-Positive Card (GP-Card - bioMeriéux). New Gram-positive cocci species have been identified from clinical specimens in recent years, and these species exhibit phenotypic profiles similar to other staphylococcal strains ${ }^{13}$. Although these species were not included in our study, the tested strains allowed us to evaluate the accuracy of the automated system in identifying the common species isolated in the clinical setting.

Our data indicated that the Vitek 2 system provided inaccurate susceptibility test results for oxacillin and vancomycin, as the agreement rate with the reference method was very low, and the error rates, mainly VMEs and MEs, were higher compared to other studies ${ }^{14}$. Although our study has evaluated the antimicrobial susceptibility of commonly isolated strains, such as $S$. aureus and $E$. faecalis, most of the isolates were microorganisms that belong to coagulase-negative staphylococci (CoNS) and non-faecalis enterococci. A previous meta-analysis reported that the discordant results in bacterial identification could be explained by the metabolic rate of the bacterial isolates, as slow metabolism can lead to ambiguous reactions during the short incubation times used by the automated instruments ${ }^{15}$. These characteristics of the samples could interfere with the AST automated method, as the staphylococci and enterococci isolates exhibited very major errors.
In conclusion, the Vitek 2 Compact system software version 5.03 correctly identified the commonly isolated Gram-positive cocci; however, the limitations of the method may lead to ambiguous findings and the inability to identify uncommon microorganisms. Therefore, additional phenotypic tests may be necessary to identify some strains at the species level. Additionally, critical inquiry of the AST results reported by the automated method showed discrepancies in the antimicrobial susceptibilities that might occur in uncommon isolated pathogens.

\section{ACKNOWLEDGMENTS}

We are thankful to bioMeriéux for the opportunity to work with the Vitek 2 Compact system technology.

\section{CONFLICT OF INTEREST}

The authors declare that there is no conflict of interest.

\section{FINANCIAL SUPPORT}

This work was supported by Conselho Nacional de Desenvolvimento Científico e Tecnológico (CNPq) and Fundação de Amparo à Pesquisa do Estado do Rio Grande do Sul (FAPERGS) (Edital Programa de Apoio a Núcleos Emergentes - PRONEM 03/2011 number 11/2058-5).

\section{REFERENCES}

1. Landrum ML, Neumann C, Cook C, Chukwuma U, Ellis MW, Hospenthal DR, et al. Epidemiology of Staphylococcus aureus blood and skin and soft tissue infections in the US military health system, 2005-2010. JAMA 2012; 308:50-59.

2. Wallet F, Loïez C, Renaux E, Lemaitre N, Courcol RJ. Performances of VITEK 2 colorimetric cards for identification of gram-positive and gramnegative bacteria. J Clin Microbiol 2005; 43:4402-4406.

3. Bannerman TL, Peacock SJ. Staphylococcus, Micrococcus and other catalase-positive cocci. In: Murray PR, Baron EJ, Jorgensen J, Pfaller M, Landry ML, editors. Manual of Clinical Microbiology. Washington, D.C: ASM; 2007.

4. Teixeira LM, Carvalho MGS, Facklam RR. Enterococcus. In: Murray PR, Baron EJ, Jorgensen J, Pfaller M, Landry ML, editors. Manual of Clinical Microbiology, Washington, DC: ASM; 2007. 
5. Clinical and Laboratory Standards Institute (CLSI). Methods for Dilution Antimicrobial Susceptibility Tests for Bacteria That Grow Aerobically; Approved Standard - Sixth edition. document M07-A6. Wayne, PA: CLSI; 2003.

6. Clinical and Laboratory Standards Institute (CLSI). Performance standards for antimicrobial susceptibility testing - Twenty-first informational supplement. document M100-S21 Wayne, PA: CLSI; 2011.

7. Chatzigeorgiou KS, Siafakas N, Petinaki E, Argyropoulou A, Tarpatzi A, Bobola M, et al. Identification of staphylococci by Phoenix: validation of a new protocol and comparison with Vitek 2. Diagn Microbiol Infect Dis 2010; 68:375-381.

8. Barros EM, Ceotto H, Bastos MC, Santos KR, Giambiagi-Demarval M. Staphylococcus haemolyticus as an important hospital pathogen and carrier of methicillin resistance genes. J Clin Microbiol 2012; 50:166-168.

9. Arslan F, Saltoglu N, Mete B, Mert A. Recurrent Staphylococcus warnerii prosthetic valve endocarditis: a case report and review. Ann Clin Microbiol Antimicrob 2011; 23:14.

10. d'Azevedo PA, Antunes AL, Martino MD, Pignatari AC. Staphylococcus cohnii spp urealyticus: case report on an uncommon pathogen. Rev Soc Bras Med Trop 2008; 41:197-199.
11. d'Azevedo PA, Siquiera I, Gugel J, Antunes AL, Secchi C, Pasternak J, et al. Evaluation of the automated system Vitek 2 for identification and antimicrobial susceptibility testing of Brazilian Gram-positive cocci strains. Braz J Infect Dis 2009; 13:107-110.

12. Arias CA, Murray BE. The rise of the Enterococcus: beyond vancomycin resistance. Nat Rev Microbiol 2012; 16:266-178.

13. d'Azevedo PA. Comin G, Cantarelli V. Characterization of a new coagulase-negative Staphylococcus species (Staphylococcus pettenkoferi) isolated from blood cultures from a hospitalized patient in Porto Alegre, Brazil. Rev Soc Bras Med Trop 2010; 43:331-332.

14. Gherardi G, Angeletti S, Panitti M, Pompilio A, Di Bonaventura G, Crea F, et al. Comparative evaluation of the Vitek-2 Compact and Phoenix systems for rapid identification and antibiotic susceptibility testing directly from blood cultures of Gram-negative and Gram-positive isolates. Diagn Microbiol Infect Dis 2012; 72:20-31.

15. Chatzigeorgiou KS, Sergentanis TN, Tsiodras S, Hamodrakas SJ, Bagos PG. Phoenix 100 versus Vitek 2 in the identification of gram-positive and gram-negative bacteria: a comprehensive meta-analysis. J Clin Microbiol 2011; 49:3284-3291. 